\title{
USE OF CFD FOR THERMAL COUPLING IN AEROENGINE INTERNAL AIR SYSTEMS APPLICATIONS
}

\author{
Zixiang Sun* ${ }^{1}$, John W. Chew ${ }^{2}$ and Nicholas J. Hills ${ }^{3}$ \\ ${ }^{* 1}$ Thermo-Fluid Systems UTC, School of Engineering, University of Surrey \\ Guildford, Surrey, GU2 7XH, UK \\ Tel: +44-1483-682333 / FAX: +44-1483-686611 \\ E-mail: Zixiang.Sun@surrey.ac.uk \\ 2 Thermo-Fluid Systems UTC, School of Engineering, University of Surrey \\ Guildford, Surrey, GU2 7XH, UK \\ ${ }^{3}$ Thermo-Fluid Systems UTC, School of Engineering, University of Surrey \\ Guildford, Surrey, GU2 7XH, UK
}

\begin{abstract}
With the rapid progress of computational fluid dynamics (CFD) and computer technology, CFD has been increasingly used for aero-engine component temperature predictions. This paper presents a review of the latest progress in this aspect with emphasis on internal air system applications. The thermal coupling methods discussed include the traditional finite element analysis (FEA), the conjugate heat transfer, FEA/CFD coupling procedure and other thermal coupling techniques. Special attention is made to identify the merits and disadvantages between the various methodologies. Discussion is further extended on the steady and transient thermal coupling applications.
\end{abstract}

Keywords: thermal coupling, computational fluid dynamics, finite element analysis, internal air systems

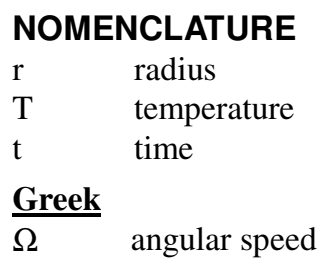

\section{INTRODUCTION}

Fast and accurate prediction of component metal temperature is one of the key issues in engine design process. With the rapid progress of CFD capability and computer power, CFD has been increasingly used to assist and to improve the metal temperature prediction in addition to the widely used finite element analysis (FEA). There are broadly three types of techniques in using CFD solutions for solid/fluid heat transfer simulations. One is generally called "conjugate heat transfer analysis", the second "noncoupled FEA/CFD procedure" and the third one "coupled
FEA/CFD approach". These are hereafter referred as conjugate analysis, non-coupled procedure and coupled approach, respectively. A good review on the thermal coupling development was made by Dixon et al in 2004. This was followed by a recent brief discussion on the issue by Chew and Hills in 2007. In the present paper, a more comprehensive review was made with emphasis on the latest progress in the internal air system applications in the recent years.

\section{CONVENTIONAL FEA METHOD}

Traditionally in industry, finite element analysis (FEA) is routinely used to predict metal temperatures in engine design. The thermal boundary conditions needed for FEA simulations are provided by thermocouple measurements and/or empirical correlations. The practice is still widely used in industry today. A latest innovative application of the technique was reported by Benito et al. (2008) at the ASME Turbo Expo 2008. Generally speaking, a FEA 
simulation could be obtained in minutes, which fits well in time scale in the design process. However, the limitation of this practice is obvious. Its effectiveness is subject to availability and applicability of the current database and correlations for a new design. A good example in comparing the traditional FEA prediction with the conjugate analysis was recently demonstrated by Starke et al (2008), showing FEA was good for global assessment, but struggled in complex geometry applications.

\section{CONJUGATE ANALYSIS}

In conjugate analysis, the solid/fluid heat transfer calculation may be realized by expanding the CFD capability to include heat conduction calculation in solid regions neighboring the fluids. Examples of such expanded CFD solvers for the conjugate analysis are NASA GlennHT code by Rigby and Lepicovsky (2001), and Aachen's CHTflow solver by Bohn et al. (2001). A number of papers have been published showing application of the conjugate analysis for engine component temperature predictions, such as a real turbine rotor-stator system simulation by Okita and Yamawaki (2002), a blade film cooling prediction by Bohn et al. (2003) and an internally cooled turbine blade application by Kusterer et al. (2004). The latest developments and applications of the conjugate analysis were reported recently by Alizadeh et al (2008) for high pressure (HP) turbine firtrees, Davison et al (2008) for an automated analysis of turbine blade cooling simulation and Okita (2006) for a simple transient simulation of a turbine disc rotor-stator rig.

The applications of the conjugate analysis were found to be limited to steady and simple transient calculations. Generally speaking, a conjugate analysis using an expanded CFD code is computationally expensive. This would be especially true for a time accurate calculation of a flight cycle, as a relatively very small time step has to be used to resolve the flow unsteadiness. A time scale in months was reported for a simple transient simulation of a rotor-stator rig (Okita, 2006). Therefore, the computational cost of performing a transient conjugate flight cycle analysis with an unsteady CFD solution for an engine application is prohibitive.

Another disadvantage of the conjugate analysis is difficult to provide further functionality of stress analysis. A latest attempt in adapting the finite volume method (FVM) for stress analysis was reported by Davison et al (2008). However, the accuracy and stability of such an adaptation for stress analysis are still questionable, compared with the matured FEA method.

\section{NON-COUPLED PROCEDURES}

Non-coupled procedures alleviate the CFD cost, where only a limited number of steady CFD calculations are performed at key engine operating conditions to produce a set of CFD based correlations, which eventually provide the necessary thermal boundary conditions for the traditional FEA calculation. Examples are two turbine disc cavity applications by Lewis and Provins (2004) and Alizadeh et al. (2007). This technique has received much attention these years. However, successful application of the non-coupled procedure is very much dependent on users' experience and expertise, such as boundary segment partitioning for the discrete correlations, and scaling of the correlations between the engine operating conditions.

\section{COUPLED APPROACH}

Coupled FEA/CFD analysis is an alternative technique, where separate FEA and CFD codes are used for solid and fluid regions, respectively, with a smooth exchange of information between the two codes to ensure continuity of temperature and heat flux. There are a variety of approaches in implementing the coupled FEA/CFD analysis. For instance, Heselhaus et al. (1992) demonstrated a 3D FEA to 3D CFD coupling procedure for cooled turbine blade application. Li and Kassab (1994) described a coupled Finite Volume Method/Boundary Element Method (FVM/BEM) approach with application to turbine blade calculation. Bohn et al. (1995) reported their coupled procedure for film-cooled turbine blade applications. Recently, Illingworth et al. (2005) reported a well established procedure coupling an in-house FEA code to a commercial CFD code, and successfully applied the procedure to turbine disc cavity calculations for flight cycle simulations. This followed work on steady state coupling by Mirzamoghadam and Xiao (2002), and Verdicchio et al. (2001). The latest extensions to the work of Illingworth et al. (2005) and validations were completed by Sun et al (2008). As a result, in this "FEA/CFD coupling" approach, an in-house FEA solver is coupled to two CFD codes, either a commercial CFD software or an in-house CFD code, which provides choice for users. A plugin is designed to exchange information between the FEA and CFD calculations to ensure the continuity of temperature and heat flux across the FEA/CFD boundaries. A thermal coupling simulation is realized through an iterative procedure between the FEA and CFD calculations. Convergence of the coupling is recognized when a pre-set criterion is met in terms of a required tolerance between two successive intermediate thermal coupling solutions. Convergence of the FEA and CFD calculations in the iterative loop is satisfied as in normal individual FEA and CFD simulations, respectively. The major features of the "FEA/CFD coupling" approach are described in the following sections.

\section{MULTIPLE CFD DOMAIN STRATEGY}

Within a FEA model, one or more CFD domains can be set up to fit in local geometry as appropriate. They may cover part or the whole of the FEA model boundary. The FEA/CFD coupling approach provides coupling capability 
for the CFD/FEA boundaries, while the rest of FEA model is still simulated using conventional thermal boundary conditions provided by thermocouple measurements and/or empirical correlations. This practice ensures great flexibility and choice for users.

\section{STEADY CFD APPROXIMATION}

For a transient flight cycle, the FEA calculations in the thermal coupling must be unsteady to reproduce the relatively slow response of metal heat conduction to a change in operating conditions of engine. Compared to this, the fluid flow time scales are much shorter, as they are determined by the fast convection of the flow. As a result, the flow may be assumed to adjust instantaneously to changes in the flow boundary conditions, and the influence of unsteadiness of fluid flow may be expected to be negligible. Therefore, steady CFD calculations could be employed. This approximation can result in significant saving in computing time for the FEA/CFD thermal coupling, as it avoids expensive unsteady CFD simulation in fluid regions and allows much larger time steps for unsteady FEA simulation of the metal heat conduction in solid regions, which means fewer time steps are needed to resolve a given transient cycle.

\section{MULTIPLE CFD MODEL FACILITY}

In the FEA/CFD thermal coupling approach, the FEA/CFD coupling approach is designed to allow a set of CFD models to be defined at key time points/conditions in the transient cycle to represent "steady" operating conditions, such as idle, maximum take-off (MTO) and cruise conditions. For each "steady" operating condition, a CFD solution is obtained by solving the corresponding CFD model. For a transient operating condition, such as engine acceleration and deceleration, a CFD solution is obtained, as an approximation, by a linear interpolation of two corresponding CFD solutions obtained at its neighboring "steady" operating conditions. To speed up the CFD calculations, initial CFD solutions are prepared in advance for each CFD model, assuming CFD wall boundaries being temporarily adiabatic. The CFD solution obtained with dynamically updated boundary conditions at a time step is always used as initial flow field for the next. As the difference of CFD solutions between two successive time steps is expected to be small, fewer iterations are needed for each CFD solution. All these contribute to further saving in computational time.

\section{“ENERGY EQUATION ONLY" OPTION}

Normally, a CFD solution is obtained by solving all the governing equations of fluid flow. This type of solution is hereafter referred as "full equations" option. As an alternative to the "full equations" option, the "energy equation only" option is developed, where energy equation only is solved during the thermal coupling, and the corresponding flow field is frozen, i.e., re-solution of flow field is bypassed. This further approximation can produce extra saving in the computational time.

The "energy equation only" option has been proven very useful in many applications. It has long been recognized that there are situations where fluid properties are essentially independent of temperature and the flow energy equation has no influence on the flow field. In this case, the flow energy equation is linear in temperature. Chew et al (1996) made use of this in their coupled CFD/FEA thermal solution for a turbine blade. This option was also demonstrated with good results by Sun et al (2008) for a rotor-stator example and two engine rig test cases.

\section{PARALLEL COMPUTATION}

For a mediate and large thermal coupling problem, parallel computation is always a wise option to reduce the wall clock time. In the "FEA/CFD coupling" approach, parallel computation has been implemented on both UNIX and LINUX systems. Satisfactory parallel computation performance was demonstrated on PC cluster platforms.

\section{COUPLING EXAMPLE}

The described "FEA/CFD coupling" approach has been successfully applied to a number of test cases with satisfactory results. For the demonstration purpose, only a 4 stage low pressure (LP) turbine disc cavity was given here. The geometry of the FEA and CFD models are shown in Figure 1. The area highlighted by red lines encloses the CFD domain, and FEA/CFD thermal coupling takes place through these boundaries. Both FEA and CFD models are axisymmetric. Three key dimensions are also shown in this figure for reference.

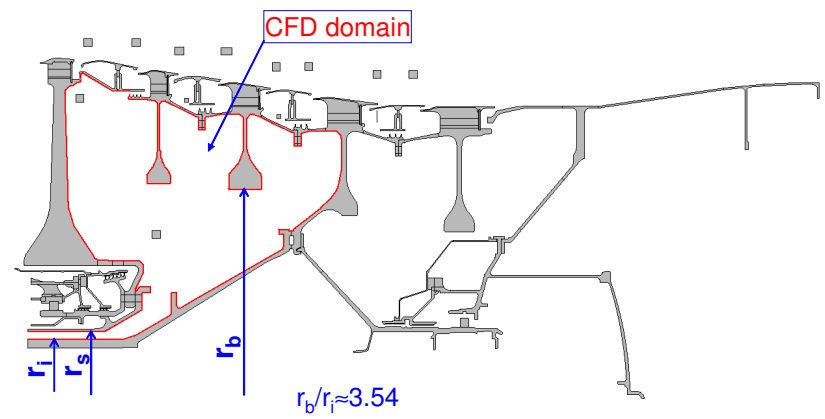

Fig.1 FEA and CFD models for a LP turbine cavity

The simulated transient cycle in terms of the LP turbine disc angular speed $\Omega$ versus time is given in Figure 2. It can be seen that the transient cycle covers a typical range of operating conditions from idle, engine acceleration to maximum take-off (MTO). All the flows were simulated with the standard $\mathrm{k}-\varepsilon$ turbulence model, as the both 
rotational and inflow Reynolds numbers for both the idle and MTO conditions were estimated to be high enough to justify a turbulent flow simulation.

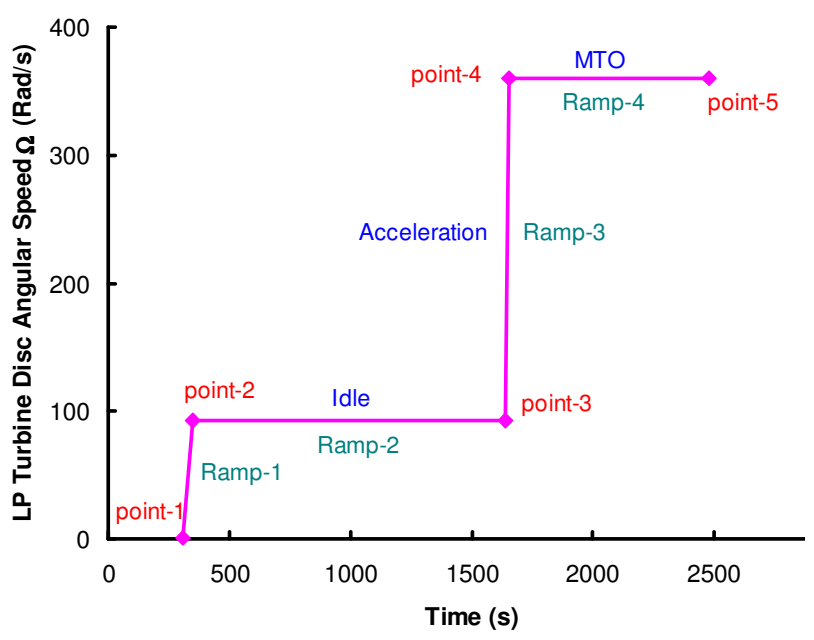

Fig.2 Transient thermal cycle for the LP turbine cavity

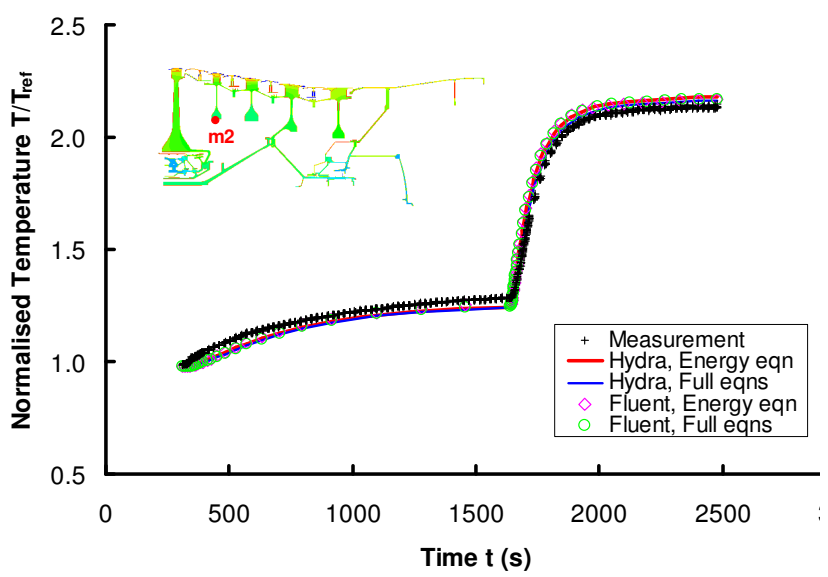

Fig.3 Comparison of temperature history at point $\mathrm{m} 2$

Figure 3 shows a comparison of the temperature histories at a typical monitoring point $\mathrm{m} 2$, obtained using both "energy equation only" and "full equations" options. The metal temperature contours obtained at the end of the thermal coupling transient cycle are also given in this figure for an overall picture of temperature distribution at $\mathrm{t}=2477 \mathrm{~s}$. For reference, the thermocouple data provided by Rolls-Royce plc were also plotted, together with coupling results using the FLUENT CFD code. In Figure 3, the red and blue solid lines indicate the results obtained using Hydra CFD solutions with "energy equation only" and "full equations" options, respectively. The black plus signs denote the Rolls-Royce thermocouple measurements. The pink diamonds and green circles designate their corresponding counterparts obtained using FLUENT. It can be seen that agreement between the thermal coupling results using "energy equation only" and "full equations" options with the Hydra and FLUENT codes is very good. The agreement between the thermal coupling predictions and Rolls-Royce measurements is satisfactory, which is generally within the measurement uncertainty.

With regard to the computational cost, it was reported that all coupling simulations conducted so far finished within 25 hours in terms of wall clock time (Sun et al 2008). The timings were obtained on a PC-cluster cluster node with a $2.4 \mathrm{GHz}$ Xeon CPU. Use of the "energy equation only" option was proven helpful in reducing the computing time. The speedup obtained so far is up to 3.1. Obviously, the speedup is case dependent. The speedup is defined as a ratio of the wall-clock time consumed between using the "energy equation only" option and its corresponding "full equations" one.

\section{CONCLUDING REMARKS}

A comprehensive review on the solid/fluid thermal coupling techniques was conducted with emphasis on the internal air system applications. It is believed the traditional finite element analysis (FEA) will be still a practical tool in engine design for many years to come, as it can provide useful information in minutes. Huge database and extensive experience accumulated make it very helpful to fit in the design requirements and time scale. The technique is still in evolution and more innovative applications and developments of the technique can be expected.

Conjugate heat transfer analysis is expensive, although significant progress has been achieved in this area. Its application will be mainly limited to steady simulations for foreseeable years. Its disadvantage in the stress analysis capability is also a major challenge to become a design tool in industry.

The non-coupled procedures are very useful as long as the technique is used wisely with experience and expertise. More application and development in the technique can be expected. However, the usage of technique is believed to be limited for many years to come until a standardized and automated procedure of procedure is developed.

Coupled approaches take advantages of two separate codes, i.e. FEA for solid domain and CFD for fluid regions, respectively. Both metal temperature prediction and stress analysis can be conducted simultaneously through FEA when required. The FEA/CFD coupling approach developed by Illingworth et al (2005) and further extended by Sun et al (2008) demonstrated good results for engine applications with encouraging computational efficiency. The "energy equation only" option has been proven useful with good speedup. More improvements were reported under way to include an enhancement in computational 
efficiency, automation of the CFD model and mesh generation, and adaptation of displacement and deformation from "cold" to "hot" engine operating conditions. The technique can be integrated with $\mathrm{CAD}$ to speed up the investigation of effect of geometry alteration, metal temperature prediction and stress analysis, tip clearance simulation in the design process. The whole process could be achieved in weeks and possibly in days in future. It is very promising to become a part of design tool set to be used in industry.

\section{ACKNOWLEDGMENTS}

Funding from the Department of Trade and Industry (DTI) and Rolls-Royce plc is gratefully acknowledged.

\section{REFERENCES}

Alizadeh, S., Mabilat C., Jackson D. and Clarkson R., 2008, "Conjugate Heat transfer study of a biaxial rig: application to the lifing of HP turbine disc firtrees", Proc. ASME Turbo Expo 2008, Paper no. GT2008-51297, pp. 113. June 9-13, 2008, Berlin Germany

Alizadeh, S., Saunders, K., Lewis, L.V. and Provins, J, 2007, "The Use of CFD to Generate Heat Transfer Boundary Conditions for a Rotor-Stator Cavity in a Compressor Drum Thermal Model", GT2007-28333, ASME Turbo Expo 2007, May 14-17, 2007, Montreal, Canada.

Benito, D., Dixon, J and Metherell, P. 2008, "3D Thermo-mechanical modeling method to predict compressor local tip running clearances", Proc. ASME Turbo Expo 2008, Paper no. GT2008-50780, pp. 1-10. June 9-13, 2008, Berlin, Germany.

Bohn, D., Bonhoff, H., Schonenborn, H. and Wihelmi, H., 1995, "Validation of a Numerical Model for the Coupled Simulation of Fluid Flow and Adiabatic Walls with Application to Film-Cooled Turbine Blades", VDIBerichte 1186, pp259-272.

Bohn, D., Kruger, U. and Kusterer, K., 2001, "Conjugate Heat Transfer: An Advanced Computational Method for the Cooling Design of Modern Gas Turbine Blades and Vanes", Heat Transfer in Gas Turbine, eds. Sunden B. and Faghri M., pp. 58-108, WIT Press, Southampton, UK.

Bohn, D., Ren, J. and Kusterer, K., 2003, "Conjugate Heat Transfer Analysis for Film Cooling Configurations with Different Hole Geometries", ASME 2003-GT-38369.

Chew, J.W. and Hills, N.J., 2007, "CFD for Turbomachinery Internal Air Systems", Philosophical transactions of the Royal Society (Series A), Aerospace CFD Theme Issue.
Chew, J. W.; Taylor, I. J.; Bonsell, J. J., 1996, "CFD developments for turbine blade heat transfer", IMECHE CONFERENCE TRANSACTIONS - 1996 ; VOL 1 ; Pages: 51-64.

Davison J.B., Ferguson S. W., Mendonca, F.G., Peck A. F. and Thompson, A., 2008, "Towards an automated simulation process in combined thermal, flow and stress in turbine blade cooling analysis", Proc. ASME Turbo Expo 2008, Paper no. GT2008-51287, pp. 1-8. June 9-13, 2008, Berlin Germany

Dixon, J. A., Verdicchio, J. A., Benito, D., Karl, A. and Tham, K. M., 2004, "Recent developments in gas Turbine component temperature prediction methods, using computational fluid dynamics and optimization tools, in conjunction with more conventional finite element analysis techniques", Proc. Instn Mech. Engrs, Vol. 218, Part A; J. Power and Energy, pp. 241-255.

Heselhaus, A., Vogel, D.T. and Krain, H, 1992, "Coupling of 3D-Navier-Stokes External Flow Calculations and Internal 3D-Heat Conduction Calculations for Cooled Turbine Blades", AGARD, p40.1-40.9.

Illingworth, J.B., Hills, N. J. and Barnes, C.J., 2005, "3D Fluid-Solid Heat Transfer Coupling of an Aero Engine Pre-Swirl System", GT2005-68939, ASME Turbo Expo 2005, June 6-9, 2005, Reno-Tahoe, Navada, USA.

Kusterer, K., Bohn, D. Sugimoto, T. and Tanaka, R., 2004, "Conjugate Calculations for a Film-Cooled Blade under Different Operating Conditions", ASME 2004-GT53719.

Lewis, L. V. and Provins, J. I., 2004, “A Non-Coupled CFD-FE Procedure to Evaluate Windage and Heat Transfer in Rotor-Stator Cavities", ASME GT2004-53246, ASME Turbo Expo 2004, June 14-17, 2004, Vienna, Austria.

Li, H. and Kassab, A. J., 1994, "A Coupled FVM/BEM Approach to Conjugate Heat Transfer in Turbine Blades", AIAA paper 94-1981.

Mirzamoghadam, A.V., and Xiao, Z., 2002, "Flow and Heat Transfer in an Industrial Rotor-Stator Rim Sealing Cavity", ASME Journal of Engineering for Gas Turbines and Power, Vol. 124, pp125-132, 2002.

Okita, Y.., 2006, "Transient thermal and flow field in a turbine disk rotor-stator system", Proc. ASME Turbo Expo 2006, Paper no. GT2006-90033, pp. 1-11. May 8-11, 2006, Barcelona, Spain

Okita, Y. and Yamawaki, S. 2002, "Conjugate Heat Transfer Analysis of Turbine Rotor-Stator Systems”, ASME 2002-GT-30615.

Rigby, D. L. and Lepicovsky, J., 2001, "Conjugate Heat Transfer Analysis of Internally Cooled Configurations", ASME 2001-GT-0405.

Starke C. and Janke E., Hofer T. and Lengani D., 2008, "Comparison of a conventional thermal analysis of a 
turbine cascase to a full conjugate heat transfer computation", Proc. ASME Turbo Expo 2008, Paper no. GT2008-51151, pp. 1-11. June 9-13, 2008, Berlin Germany.

Sun, Z., Chew J.W., Hills, N.J., Volkov, K. N. and Barnes, C. J., 2008, "Efficient FEA/CFD Thermal coupling for Engineering Applications"”, Proc. ASME Turbo Expo 2008, Paper no. GT2008-50638, pp. 1-11. June 9-13, 2008, Berlin Germany.

Verdicchio, J.A., Chew, J.W., and Hills, N.J., 2001, "Coupled Fluid/Solid Heat Transfer Computation for Turbine Discs", ASME paper 2001-GT-0123. 
